53 郭开仲. 用计算机处理不相容问题. 智囊与物元分析, 1986, 2: 41 48

54 陈朝春, 林复华. 可拓语言的研究方向与展望. 智囊与物元分析, 1986, 3: 40 41

55 王万良, 赵燕伟. 探索机械智能 CAD 系统的可拓决策方法. 系统工程理论与实践, 1998, 2: 114 117

56 刘 巍. 物元神经网络. 见: 蔡 文主编. 从物元分析到可拓学(论文集). 北京: 科学技术文献出社, 1995, 311 317

57 杨国为. 物元动态系统分析. 青岛: 青岛出版社, 1997

(1998-11-02 收稿, 1999-02-03 收修改稿)

\title{
瑞利光散射及其在生物化学分析 中的应用研究
}

马春琪 刘 瑛 李克安“童沈阳

(北京大学化学与分子工程学院, 北京 100871.* 联系人)

摘要 对瑞利(Rayleigh) 光散射的理论基础及其在生物化学分析中的应用进行了较全面的介 绍. 其中讲述了共振增强的瑞利光散射和普通瑞利光散射之间的联系和区别，它们在生物大 分子测定方面的应用以及两者的特点和测定过程中的影响因素等. 通过比较瑞利光散射和二 级/ 反二级瑞利光散射, 指出了瑞利光散射的测定优势. 最后对光散射技术提出了一些见解 并对其研究的意义和发展前景做出了一些评价和展望.

\section{关键词 瑞利光散射 共振光散射 蛋白质 核酸}

光散射是指一束光线通过介质时在入射光以外的方向上观测到光强的现象，它是电磁辐 射与物质间相互作用的一种表现形式.

分子在吸收能量较低的光子之后并不足以使分子中电子跃迁到电子激发态，而只使电子 激发至基态中较高的振动能级. 在较短的时间里(约 $10^{-12} \mathrm{~s}$ ), 电子将返回原来的能级，并伴 随着在不同方向上发出瑞利光散射( RLS), 瑞利光散射是一类入射光与反射光波长相同的光 散射. 当分子在返回基态时并没有返回至原来的能级, 而返回到稍高的或稍低的能级, 引起 的散射光称为 Raman 光. 瑞利散射光和 Raman 光对荧光分析有显著的影响, 因而常常成为荧 光分析方法灵敏度的主要限定因素 ${ }^{[1]}$

早已发现, 散射光并不是浑浊溶液的特有现象, 各向同性的透明介质也有光散射. 1910 年, 爱因斯坦( Einstern) 首先用宏观波动理论对后者进行了准确描述, 以后关于光散射理论和 应用研究的文章不断. 以前, 光散射主要用在大分子(生物大分子) 的结构及分子量测定等物 理性质方面，由于光散射实验可以提供有关粒子大小、形状等方面的信息，所以光散射技术 在胶体化学和高分子溶液研究方面有了广泛的应用. 但在分析化学领域, 散射光常常作为分 析测定中的影响因素需采取措施加以避免或减小. 自从 1993 年 Pasternack 等人 ${ }^{[2]}$ 首次用共振 光散射技术对卟啉类化合物在核酸上的聚集进行了研究之后, 揭开了光散射技术在分析化学 方面应用的序幕并使光散射技术逐步成为仪器分析方面的重要补充. 下面将评述瑞利光散射 在生物化学分析方面应用的近期进展，以及这种实验技术的特点和影响因素. 最后展望该技 术在今后的发展、应用前景, 以期对生物大分子微量测定方面的研究有所帮助. 


\section{1 瑞利光散射的理论基础及其在生物化学分析中的应用}

\section{1 瑞利光散射的理论基础}

80 年代以前, 关于瑞利光散射的理论和应用都集中在没有吸收的波长范围内, 此时光散 射强度与 $\lambda^{-4}$ 成正比. 实际上, 所有的吸收过程都与光散射紧密相连, 总有极少量的被吸收 的光又以光散射的形式发散出来, 这个现象被称为“共振增强的瑞利光散射”, 它从最初被预 见 $^{[3]}$ 到第 1 次发现 ${ }^{[4]}$ 又经历了 41 年. Miller 等人 ${ }^{[5]}$ 首先于 1978 年用宏观波动理论对最大吸 收附近的瑞利光散射进行了理论处理, 在此基础上, Anglister 和 Steinberg 进行了修正, 以包括 溶质最大吸收处的散射光 ${ }^{[6]}$, 上述理论不仅与大量实验事实相吻合 ${ }^{\left[{ }^{[1]}\right.}$, 而且为研究溶质分 子的聚集状态奠定了理论基础.

瑞利光散射理论认为: 当一束单色光通过均匀各向同性的透明介质时, 散射光的强度与 $\lambda^{-4}$ 成正比, 但当入射光接近分子的吸收带时, 散射光强度将会增强而偏离与 $\lambda^{-4}$ 的对应关系, 即产生共振增强的瑞利光散射. 散射起因于溶液的浓度涨落, 而浓度涨落直接与浑浊度 $\tau_{c}$ 相 关, $\tau_{c}$ 表示为 ${ }^{[6]}$

$$
\tau_{c}=32 \pi^{3} n^{2}\left[(\partial n / \partial c)^{2}+(\partial K / \partial c)^{2}\right] 1000 c / 3 \lambda^{4} N_{\mathrm{A}},
$$

其中 $\lambda$ 是入射光波长. $c$ 是溶质的摩尔浓度. $n$ 是介质的平均折光指数. $N_{\mathrm{A}}$ 为 Avogado 常数. $\partial n / \partial c$ 和 $\partial K / \partial c$ 分别为浓度涨落引起的折射指数的实部 (real part) 和虚部( imaginary part) 的增 量(每 $1 \mathrm{~mol} / \mathrm{L}$ 溶质), 每个粒子都有一个散射截面 $s_{\mathrm{sca}}$, 它表示各方向上光散射能量与入射光 强度之比, $\tau_{c}$ 与光散射可建立如下关系:

$$
\tau_{c}=N^{\prime} s_{\text {sca }},
$$

式中 $N^{\prime}$ 为单位体积内散射粒子数. 综合方程 (1) 和(2), 散射截面可表示为

$$
s_{\text {sca }}=K_{m}^{4}|\alpha|^{2} / 6 \pi=K_{m}^{4}\left(a_{r}^{2}+a_{i}^{2}\right),
$$

而吸收截面 $s_{\mathrm{abs}}$ 为光能量吸收率与入射光强度之比, 它仅与 $a_{i}$ 有关:

$$
s_{\text {abs }}=K_{m} a_{i},
$$

$K_{m}$ 是溶液中光的波矢量, $K_{m}=2 \pi / \lambda ; \alpha_{r}$ 和 $\alpha_{i}$ 则分别是散射体极化能力的实部和虚部. $a_{i}$ 和 $a_{r}$ 相互关联, 当在某个波长范围内 $a_{i}$ 达到最大值时, $a_{r}$ 在此区域也有异常表现, 因此, $|a|^{2}$ 在 吸收带时最大, 并导致增强的光散射.

散射体在某波长的吸收可看成是在该光谱区域极化能力的虚部达到最大值时的结果; 厚 度为 $L$ 的样品的吸光度为

$$
A=2.3^{-1} N^{\prime} s_{\text {abs }} L=K c \text {. }
$$

上述关系式(3 5) 清楚的表明了光散射与吸收过程的密切关系, 只有当吸收过程存在并 符合一定条件时, 才会因吸收能量的参与而产生特殊的瑞利光散射-共振光散射, 此时 $\partial K / \partial c=2.303 \varepsilon \lambda_{b} n / 4 \pi$. 但当介质透明时, $\partial K / \partial c$ 为零, 而产生的是没有吸收能量参与的普通 瑞利光散射. 两者的差别仅在 $\partial K / \partial c$ 项, 当测定波长 $\lambda_{b}$ 固定下来以后, $n$ 和 $\varepsilon($ 摩尔吸光系 数) 为常数, 又因 $\partial n / \partial c=-\frac{2.303 \lambda_{0}^{2}}{2 \pi^{2}} \int_{0}^{\infty} \frac{\varepsilon v}{\lambda^{2}-\lambda_{0}^{2}} \mathrm{~d} X^{[7]}$, 故可看出 $\partial K / \partial c, \partial n / \partial c$ 均只受 $\lambda$ 和 $\varepsilon$ 影响, 故也为常数, 从而 $s_{\mathrm{abs}}$ 为常数. 由公式 (5) 可知 (其中 $K$ 为常数), 样品的吸光度 $A$ 只与单 位体积内散射粒子数 $N^{\prime}$ 有关, 也即与生物大分子的浓度 $c$ 有关. 故散射强度与生物大分子 
的浓度 $c$ 成正比, 这便是瑞利光散射定量的基础.

\section{2 共振增强的瑞利光散射在生物化学分析中的应用}

1993 年 Pasternack 等人 ${ }^{[2]}$ 首次用共振光散射技术研究了卟啉类化合物在核酸上的聚集, 从而首次将该技术与化学过程联系起来. 而黄承志等人利用当卟啉在蛋白质或核酸上聚合组 装时产生的增强的共振光散射信号与生物大分子浓度间的线性关系，建立了共振光散射技术 定量测定牛物大分子的新的分析方法 ${ }^{[12,13]}$. 研究表明. 并不是任何一种聚集状态都能产生共 振光散射增强, 只有那些可以生成聚集状态、聚合粒子非常大且具有较大的摩尔吸光系数时, 才能导致共振光散射信号增强, 这就是为什么叶啉的单体和二聚体或三聚体并不显示散射增 强的原因 ${ }^{[2,14]}$. 但寻找既能与生物大分子作用、其聚集状态又能满足上述条件的物质非常不 易，故而这种方法的应用受到了限制.

1.3 普通的瑞利光散射在生物化学分析中的应用

经研究发现, 一些能与蛋白质或核酸发生作用的染料小分子(这些染料小分子都没有聚合 现象)，也能使光散射信号增强 ${ }^{[15 \sim 19] 1)}$. 以甲基紫/ 50.0 小牛胸腺 DNA (ctDNA) 体系为例, 图 1 给出了其吸收 与散射谱图，与卟啉-生物大分子生成聚合物的共振 光散射体系(其散射峰与吸收峰的位置重合) 不同 ${ }^{2)}$, 对于染料小分子与生物大分子作用的光散射体系, 其吸收峰与散射峰有很好的倒映关系. 由图 1,2 可 见, 蛋白质或核酸的加入，使光散射信号明显增强, 但并不改变光散射谱图的形状, 这是由于所用的生 物大分子的浓度极低, 以至于它的加入不影响染料 吸收谱图的形状和吸光度值, 故而也不影响散射谱 图的形状. 这种光散射我们暂称之为一种普通的瑞 利光散射 $\left(\lambda_{\mathrm{ex}}=\lambda_{\mathrm{em}}\right)$, 而共振光散射可以说是一种特 殊的瑞利光散射，两者虽在某些方面有相似之处但 产生机制有较大差别.

瑞利光散射增强并不是由小分子在蛋白质/核 酸上聚合产生的. 因所用染料及染料-生物大分子复 合物的摩尔吸光系数都不大于 $10^{5} \mathrm{~L} \cdot \mathrm{mol}^{-1} \cdot \mathrm{cm}^{-1}$ $(\lambda=500 \mathrm{~nm})$, 而即使对于具有最大摩尔吸光系数达 到这一数量级的生色团, 重新作为散射光发射的那

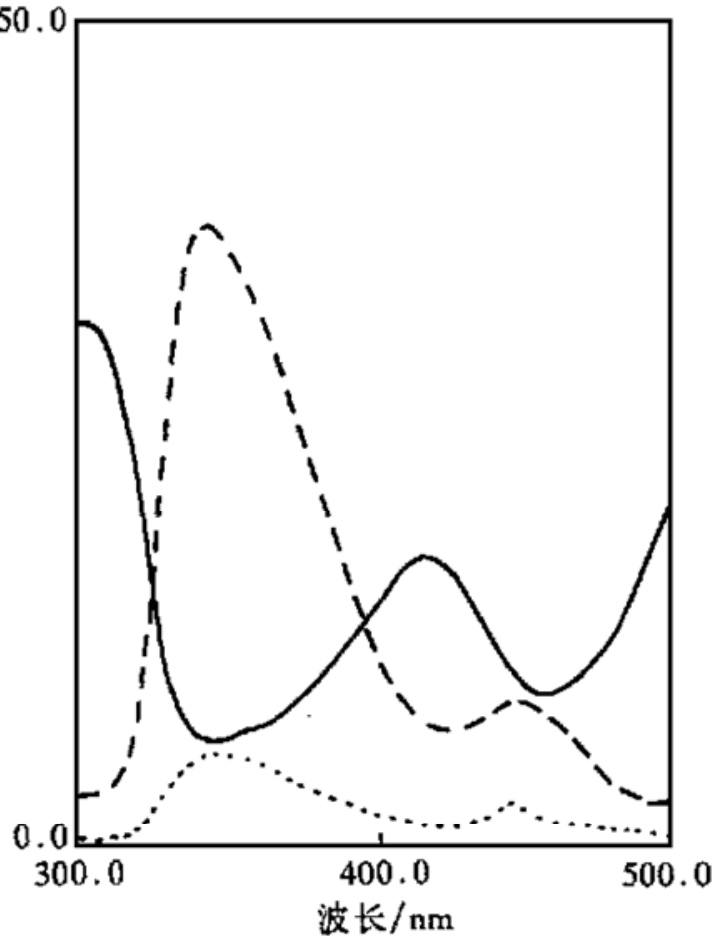

图 1 甲基紫的吸收和散射谱图 ——为甲基紫的吸收谱图; ......为甲基紫的 RLS 谱 图; ----为甲基紫/ ctDNA 结合的 RLS 谱图. 测定条 件: 纵坐标 $\times 1$ 部分吸收仅占 $4 \times 10^{-6}$, 此种情况下, 染料的散射光强度比溶剂的散射光低 1 2 个数量级, 故 很难甚至根本不可能检测到增强的散射信号，即不能观测到共振光散射。因此对不发生聚集 的小分子染料，很难检测到共振光散射信号，但对于有色团聚集体, 这种散射增强相当显著, 这是因为吸收和散射对聚合体的体积大小的依赖关系不同. 对含一定浓度生色团的溶液, 每

1) Liu Y, Ma C Q, Li K A, et al. A simple and sensitive assay for nucleic acids by rayleigh light scattering technique with rhodamine B. Anal Chim Acta (accepted)

2) 黄承志. 北京大学化学与分子工程学院. 北京大学博士论文, 1996: 77 81 

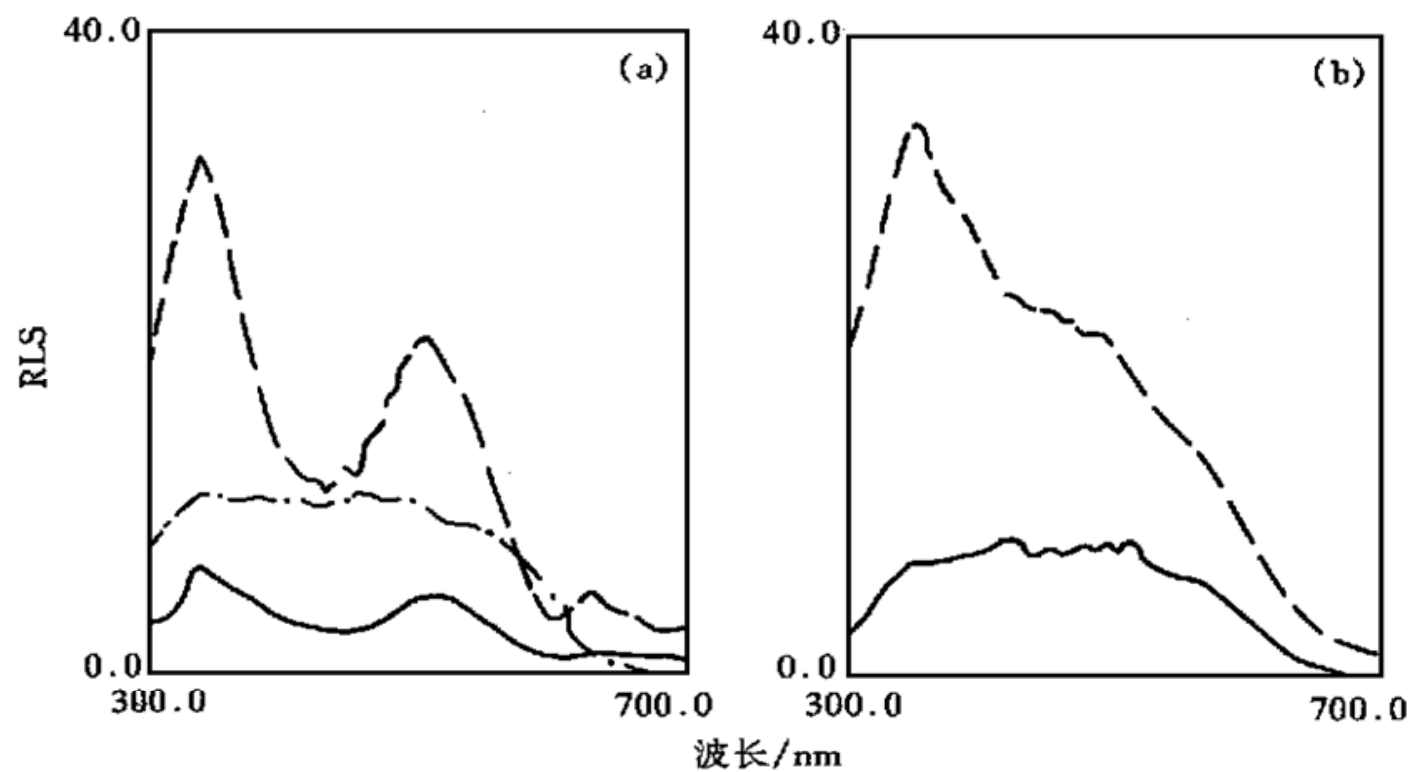

图 2 染料在 BSA 存在 $(--)$ 、空白 $(-\longrightarrow)$ 和纯水 $(-\rightarrow-)$ 时的 RLS 谱图 染料: (a) 四碘酚磺酞; (b) 茜素紫

个球体的吸收与球体的体积成正比, 而单位体积内球体的个数与球体体积成反比, 即 $N^{\prime}$ 和 $a$ i 的乘积保持不变, 故吸光度与散射球的大小无关. 另一方面, 每个球体的散射光也与球的体积 的平方成正比, 既然球体密度与其体积成反比, 因此散射光强直接正比于每个球的体积, 故聚 集体越大, 散射越强, 即使很稀浓度的集合体粒子, 共振光散射信号仍十分灵敏. 而对于普通 的瑞利光散射, 散射光强也正比于每个球的体积, 但因太强的分子吸收意味着较弱的共振光 散射增强, 加之染料本身不发生聚合, 故在染料的最大吸收波长处观察不到共振光散射信号. 然而当染料结合到生物大分子上以后，生物大分子对染料起到了富集作用，使其上染料浓度 远大于溶液中游离的染料浓度, 相当于聚集体的生成, 使散射球体积增大, 所以尽管吸收谱图 无变化, 但却观测到增强的 RLS 信号. 图 2(a) 还给出了纯水的瑞利光散射谱图, 因无吸收的 影响, 光散射信号近似与 $\lambda^{-4}$ 成正比, 异常值是由仪器波动造成的. 波长约 $320 \mathrm{~nm}$ 以下, RLS 信号随波长的降低而急剧下降. 这是由于仪器本身的灯/检测器的效率随波长紫移而迅速减小 的缘故. 比较染料与纯水的散射谱图, 可以认为染料的 RLS 谱图是在无色溶液的散射背景 上, 由于染料的吸收而产生的, 实际为受染料吸收影响的无色透明溶液的散射谱图, 吸收越 强, 散射越弱. 这样一来, 就很容易理解吸收谱图与散射谱图间的倒映关系. 对于测定酸度 下, 在 300 $700 \mathrm{~nm}$ 无吸收的染料如茜素紫 (见图 2(b) ), 其 RLS 谱图与纯水相同, 符合与 $\lambda^{-4}$ 的正比关系，牛血清白蛋白 (BSA) 的加入同样能使其 RLS 增强, 加之有色溶液的 RLS 测定波 长都位于无吸收或吸收较弱的短波区 (此区域有最大散射), 故所考察的瑞利光散射实际应属 于透明介质范畴. 此时式 (1) 中的 $\partial K / \partial c$ 项为 0 , 故与共振光散射一样, 当测定波长一定时, $n$ 和 $\partial n / \partial c$ 均为常数, 生物大分子浓度 $c$ 与光散射强度有线性关系.

那么, RLS 增强是否仅来自于大颗粒的散射体生成的呢? 为此试验了在染料不存在的情 况下, 直接往纯水中加入蛋白质时对光散射的影响, 结果表明 RLS 并不随蛋白质的浓度增加 而增强, 因此可以认为蛋白质与染料间的结合是散射信号增强的必要条件. 研究表明, 共振光 散射增强与单个生色团的电性质、生色团间的电偶合程度以及由此而形成的聚集体的大小密 切相关 ${ }^{[2,14]}$. Pasternack 等人 ${ }^{[14]}$ 在研究卟啉与 DNA 的结合时认为, 卟啉在 DNA 分子表面按照 
DNA 模板的周期性进行排列时, 使生色团的密度增大, 它们间的静电作用也随之增大, 从而显 示出很大的电子离域效应, 导致散射信号增强. 当有机小分子 (无论有吸收还是无吸收) 与生 物大分子之间有强烈的静电作用时, 且其在 BSA 上的结合数目达到一定程度时, 也相当于电 子离域程度增加, 故而可观察到增强的 RLS 信号. 总结以上实验结果, 我们认为小分子 RLS 增强的 2 个必要条件是: (1) 大颗粒散射体的生成; (2) 强静电结合及大结合数所导致的高度 的电子离域化.

\section{2 瑞利光散射的影响因素}

\section{$2.1 \mathrm{pH}$ 的影响}

测定体系对溶液酸度的变化非常灵敏, 而染料本底因散射光较弱, 故受 $\mathrm{pH}$ 的影响并不是 太明显. 体系的 RLS 随 $\mathrm{pH}$ 的增加而增大至一恒定值, 随后, RLS 信号随 $\mathrm{pH}$ 的继续增加而急 剧降低, 这个现象可从 RLS 增强的两个必要条件上得到解释. 以牛血清白蛋白(BSA) 测定为 例, $\mathrm{BSA}$ 的等电点为 4. 6, $\mathrm{pH}=4.6$ 以下, $\mathrm{BSA}$ 荷正电, 易与染料阴离子静电相吸形成复合物; $\mathrm{pH}$ 的升高, 有利于染料的质子解离而带更多的负电荷, 从而促进 BSA 与染料的结合, 但也使 BSA 上的正电荷数目减小而对两者的结合产生负作用. $\mathrm{pH}$ 的这两种相反影响在 $\mathrm{pH}=4.0$ 左 右达到平衡, 此时, 染料在 BSA 上有最大结合, 电子离域程度也较高; $\mathrm{pH}=4.0$ 后, 随 $\mathrm{pH}$ 进一 步升高, 负影响占了上风, 染料从 BSA 上解离下来, 也影响到电子的离域程度, 故 RLS 降低. 同理, 对核酸测定体系, 在酸性条件下, 核酸带负电荷易于同染料阳离子静电作用形成复合物, 随 $\mathrm{pH}$ 的升高, 不利于染料结合质子而阻碍了核酸与染料的静电结合从而使 RLS 较低.

染料与蛋白质作用的最佳 $\mathrm{pH}$ 值与分光光度法测定蛋白质的酸度条件有很大出入. 例 如, ACBK 用于分光光度法测定 BSA 的最佳酸度值为 $\mathrm{pH}=2$. 4, 用 TIPSP 作分光光度探针时,

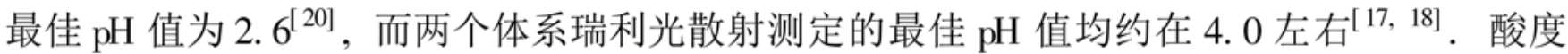
条件的较大差异与两种方法的测定机理直接相关. RLS 法主要考虑染料与蛋白质的最大结 合, 而分光光度法除要求染料分子要尽可能多地与蛋白质结合外, 还要考虑染料的两种不同颜 色型体发生转换时的 $p K_{\mathrm{a}}$ 值 ${ }^{[21]}$. 由两种测定方法都可看出, 静电引力在染料与蛋白质的结合 中起着重要作用.

另外 RLS 测定技术中, 试剂的加入顺序一般对测定都有较大影响. 若首先混合蛋白质和 染料, 因反应 $\mathrm{pH}$ 在 6 左右, 而不利于两者的结合. 所以缓冲液与染料先混合, 然后蛋白质再 加入的混合顺序, 可为蛋白质与染料的最大结合营造一个最适宜的酸度条件, 从而具有较高的 灵敏度 ${ }^{[15 ~ 18]}$. 这在染料与核酸结合的测定中也能很好的符合 ${ }^{1)}$, 从而进一步证明静电引力是 生物大分子与染料分子之间结合的主要作用力.

\section{2 生物大分子的响应差异}

前面提到, 静电引力是生物大分子与染料分子之间的主要结合力, 各蛋白质的 $-\mathrm{NH}_{3}^{+}$及 碱性氨基酸数有很大差别, 从而导致了对染料不同的 RLS 响应. 含有较多的碱性氨基酸残基 的蛋白如鱼精蛋白与染料的结合最强, 测定灵敏度也最高; 相反, 胃蛋白酶 $(\mathrm{pI}=1.0)$, 因在 测定条件下带负电荷, 故它几乎不与染料作用 ${ }^{[15 ~ 18]}$. 对若丹明 B/ 核酸结合的测定体系, RLS 还与聚合粒子的大小有关，分子量大易形成大的聚合粒子的核酸有较强的 RLS 信号 ${ }^{1)}$.

1) 见 684 页脚注 1) 


\section{3 染料浓度的影响}

染料浓度的大小影响 RLS 产率和测定的线性范围. 以蛋白质的测定体系为例，随染料浓 度的增大, 测定的灵敏度随之增大, 线性范围也随之变宽, 表明浓度增大有利于染料与蛋白质 的结合 ${ }^{[15 ~ 18]}$. 浓度太低时, 工作曲线的弯曲并不是由于染料用量不足而导致的染料在 BSA 上的饱和性结合引起的, 因为此时染料浓度仍远远大于蛋白质的浓度. 因此复合物的生成要 求必须有过量得多的染料. 另外. 测定灵敏度随染料浓度的增大而增大. 并有一个临界浓度. 高于此浓度, 测定灵敏度 (工作曲线斜率) 反而降低, 这是由于太高的染料浓度将产生较强的分 子吸收, 而这种吸收不利于散射信号的增强, 故观察到降低的 RLS 灵敏度.

\section{4 共存物质的干扰}

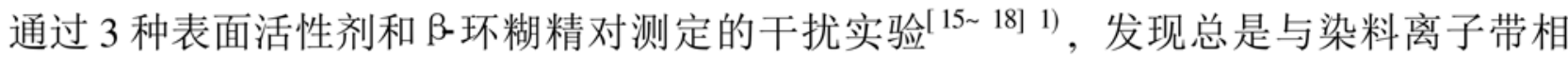
反电荷的表面活性剂阻碍生物大分子与染料间的结合, 导致本底与体系相同的 RLS 增强响 应. 这是由于带相反电荷的表面活性剂与染料离子有很强的静电作用，再次表明静电作用在 RLS 测定中的重要作用. 另外试验了金属离子、无机盐和有机物等对测定体系的影响. 总的说 来, 干扰因素不多且干扰程度不大, 又因 RLS 测定极其灵敏, 样品往往又需要成百倍乃至千倍的 稀释以迎合测定需要, 这就使得各干扰的影响更加显得微乎其微, 可根本不予考虑. 但在直接测 定尿样中的蛋白质含量时却遇到了麻烦, 是否各干扰的综合效应才是影响 RLS 测定的真正原因, 在试验 TIPSP 体系的干扰时 ${ }^{[18]}$, 各共存物质除采用一般干扰浓度外, 还试验了其 10 倍量的干扰 情况, 结果发现增大共存物质浓度后, 原来不干扰测定的物质 (主要是金属离子) 也对 RLS 信号有 明显影响, 干扰原因主要在于散射粒子数太多, 从而提高了本底溶液的光散射强度. 因此, 尽管 测定体系中, 单个共存物质浓度不是很高, 但各种共存物质的总浓度如果很大的话, 也会影响到 光散射的测定, 故对于蛋白质含量较低, 而共存物质种类和含量都很大的某些生物样品(如尿 样), 用 RLS 法直接测定其中的蛋白质含量就比较困难, 可通过标准加入法加以解决 ${ }^{[19]}$.

\section{5 工作曲线及样品测定}

考察了不同测定体系中生物大分子测定的线性范围, 与最佳实验条件一并列于 表 $1^{[15 \sim 18] 1)}$. 由表可见, RLS 法的灵敏区域正是其他常用分析方法如分光光度法难于涉足的 区域. RLS 技术的检测下限基本集中在 $0.1 \mu \mathrm{g} / \mathrm{mL}$ 左右, 这可能是由 RLS 本身的特点造成的.

表 1 瑞利光散射技术测定生物大分子的条件及结果

\begin{tabular}{clccc}
\hline 测定体系 & \multicolumn{1}{c}{ 染料浓度 } & 波长/ $\mathrm{nm}$ & $\mathrm{pH}$ & 线形范围 $/ \mu_{\mathrm{g}} \cdot \mathrm{mL}^{-1}$ \\
\hline $\mathrm{BPB} / \mathrm{BSA}$ & $0.002 \%$ & 334 & 4.0 & $0.340 \sim 18.70$ \\
$\mathrm{TIPSP} / \mathrm{BSA}$ & $0.03 \mathrm{mmol} / \mathrm{L}$ & 334 & 4.0 & $0.340 \sim 12.24$ \\
$\mathrm{Ag} 25 / \mathrm{BSA}$ & $0.0035 \%$ & 347 & 4.0 & $0.136 \sim 10.20$ \\
甲基紫/ ctDNA & $0.004 \%$ & 344 & 1.0 & $0.10 \sim 7.0$ \\
$\mathrm{RB} / \mathrm{ctDNA}$ & $0.003 \%$ & 378 & 1.0 & $0.10 \sim 16.0$ \\
\hline
\end{tabular}

共振光散射对聚集体的大小或聚集数有一定的要求, 因此对特定的生色团也有固定的检 测下限. 假设共振光散射增强为 $100 \%$, 则方程(1) 中 $\partial n / \partial c=\partial K / \partial c=\varepsilon N 4 \pi$, 进一步假设增 强的那部分浑浊度等于纯溶剂的浑浊度, 并且 $\varepsilon=1$, 在这些条件下, 将很容易观察到共振增

1) 见 684 页脚注 1) 
强信号, 浑浊度的增加量为

$$
\tau_{c}=4000 \pi n^{2} \varepsilon / 3 \lambda_{g}^{2} N_{\mathrm{A}}
$$

纯水的浑浊度在 $546 \mathrm{~nm}$ 时为 $1.6 \times 10^{-5} \mathrm{~cm}^{-1}$, 使 $\tau_{c}=1.6 \times 10^{-5}, n=1.33$, 则可得到 $\varepsilon=$ $3.9 \times 10^{6}$, 大多数染料都不具备这么大的摩尔吸光系数, 因此, 通常情况下, 观察不到增加的光 散射信号, 然而当溶质生成聚集体时, 则方程 (1) 的浑浊度须翻倍, 这个倍数即聚集数. 一般染 料的摩尔吸光系数不超过 $10^{5}$, 当 $\varepsilon=10^{5}$ 时, 聚集数为 $3.9 \times 10^{6} / 10^{5}=39$, 满足此条件即可观 察到增强的散射光.

虽然测定的 RLS 与共振光散射有很大区别, 不能生硬套用共振光散射计算聚集数的公 式, 但由于仪器本身的灵敏度所限, 散射信号增强也有一定的下限, 另外形成染料-蛋白质复合 物与聚集体的生成也确有异曲同工之处. 染料与生物大分子结合形成大的颗粒, 这些颗粒必 须在溶液中达到一定的浓度 (相当于一定的聚集数) 时, 才会产生 RLS 增强. 因染料均为小分 子, 故复合物颗粒的大小主要由生物大分子的大小决定, 对特定的分子来讲, 可能有特定的检 测下限, 这一点有待进一步证实.

\section{3 关于光散射的一些见解及前景展望}

\section{1 对光散射技术的一些见解}

与分光光度法相比, 光散射法对颗粒的大小非常灵敏, 这是其比分光光度法灵敏度高的主 要原因, 但也有不利的一面, 当有些染料与蛋白质形成的复合物在溶液中不稳定, 例如CBBG 与 BSA 的复合物在水溶液中缓慢沉淀, 如用分光光度法检测, 在 $0.5 \mathrm{~h}$ 之内, 吸光度变化幅 度不大，但 RLS 信号却非常不稳定. 故产物的稳定性也是光散射法测定的必要条件.

共振光散射确实是检测聚集体生成的一个有利工具, 但也有误区. 本文作者在结合大量

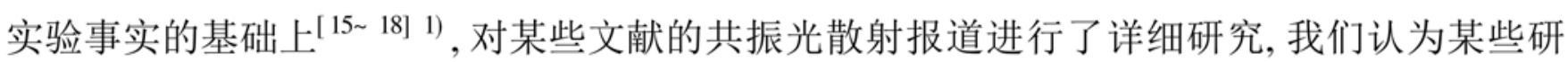
究者对共振光散射存在误解. 图 3 给出了叶绿素 a 以单体存在于丙酮中或在甲酰胺: 磷酸盐 缓冲溶液中发生聚集时的吸收和散射谱图 ${ }^{[22]}$. 图 3(a) 和( d) 两图的倒映关系与本文研究比较 符合, 在 $450 \mathrm{~nm}$ 因吸收较弱, 故而散射很强, 随后散射强度随 $\lambda$ 增大而锐减, 正好与他们所测 水的散射光随波长的变化趋势一致. 由图 3(c) 图可看到叶绿素 a 聚集态的吸收峰在 453 和 $686 \mathrm{~nm}$, Pasternack 等人认为图 3(e) 图 $458 \mathrm{~nm}$ 的散射峰反映了聚集过程中有中间产物生成, (f) 图 469 和 $699 \mathrm{~nm}$ (图中未标出) 的散射则为分别对应着图 3(c) 图中两种最终聚集体产物的共振 光散射. 比较图 3(b) 和 (e) 及 (c) 和(f) 图, 可以发现散射峰位置并不在 $\lambda_{\max }$ 处, 而在吸收足够 小的地方, 这一点与我们上述实验情况吻合, 因此, 散射峰并不是对应着聚集体最大吸收的严 格意义上的共振光散射, 而是由于聚集体本身高吸光度的影响, 在溶剂光散射背景上的普通瑞 利光散射. 相似情况也出现在 DNA 与卟啉 $t$-CuPagg 的结合上 ${ }^{[2]}$. 故我们认为当 RLS 峰不在 $\lambda_{\max }$ 处时, 仅从散射谱图并不能断言有聚合现象发生, 而需要通过其他仪器方法( 分光光度法、 CD 谱等) 来进一步验证.

除 RLS 外, 刘绍璞等人 ${ }^{[23,24]}$ 曾报道了离子缔合物的二级瑞利光散射和反二级瑞利光散 射现象, 最早将光散射技术应用到金属元素的灵敏测定方面，在测定生物大分子方面, 我们用 (甲基紫/ ctDNA) 体系比较了 RLS 和二级/ 反二级瑞利光散射两种方法的灵敏度, 由图 1, 4 比

1) 见 684 页脚注 1) 
较可见, RLS 测定的灵敏度远高于后者, 且二级/ 反二级瑞利光散射法在确定最佳波长时比较 费时费力. 从而可以看出在生物大分子测定方面, RLS 法有明显的优势.
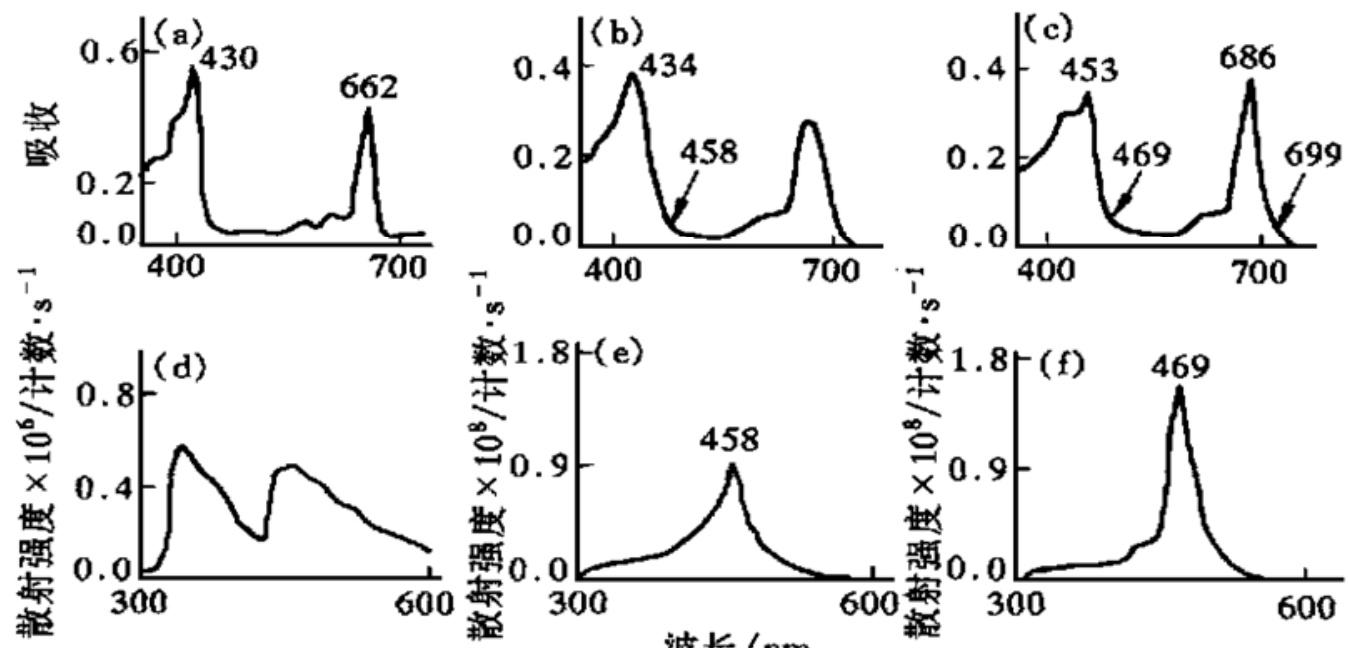

波长 $/ \mathrm{nm}$

图 3 溶液中叶绿素 $\mathrm{a}$ 的吸收和共振光散射谱图

(a) (c) 为 $5 \mu \mathrm{mol} / \mathrm{L}$ 叶绿素的吸收图谱. (a) 丙酮; (b) 甲酰胺: 磷酸盐缓冲液, 混和后 $4 \mathrm{~min} ;$ ( c) 甲酰胺: 磷酸 盐缓冲液, 混和后 $60 \mathrm{~min}$. (d) (f) 为 $5 \mu \mathrm{mol} / \mathrm{L}$ 叶绿素 a 的共振光散射谱图. (d) 丙酮; (e) 甲酰胺: 磷酸盐缓冲 液, 混和后 $7 \mathrm{~min}$; (f) 甲酰胺: 磷酸盐缓冲液, 混和后 $123 \mathrm{~min}$. 光谱未作光电倍增管响应校正

\section{2 结论及前景展望}

许多能用分光光度法测定的探针染料也有可能用于 RLS 法测定, 对于在水溶液中无颜色变化的且用通常仪 器检测不出来的探针反应，也可通过 RLS 法检测. 从而 扩大了生物大分子测定的探针范围，为蛋白质/核酸与小 分子的机理探讨提供了一个重要的研究手段.

共振光散射法已被用于分子聚集状态的研究, 它可以 为理解复合体系的化学活性、生物活性及药理活性与体系 组分的分子聚集状态的关系提供一个检测和表征 ${ }^{[25]}$ ，使 光散射技术成为化学研究的一种新兴手段.

光散射技术的测定灵敏度很高, 可达纳克级, 从而为 生物化学及临床分析样品的微量分析测定提供了一条新 的途径.

光散射分析实验只需普通的苂光分光光度计, 试剂易 得, 加之该方法操作简单易行, 故有利于对大批量样品的 测定，因此便于在各实验室中推广.

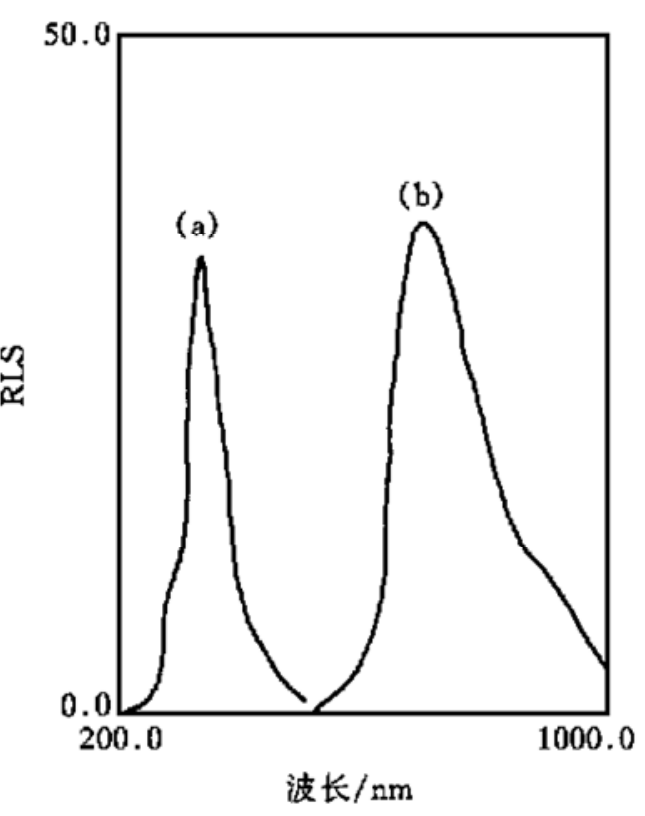

图 4 甲基紫/ ctDNA 的二级( a) 和反 二级 (b) 瑞利光散射谱图 测定条件: 纵坐标 $\times 3$, 其他条件同图 1

分析化学以其先进的设备和灵敏的分析手段而起着联系化学与生物学、物理学的纽带作 用, 而光散射法有望成为分析化学仪器方法的重要补充. 致谢 本工作为国家自然科学基金( 批准号: 29775003) 资助项目.

\section{参 考 文 献}

1 陈国珍，黄贤智，郑朱梓，等. 苂光分析法(第二版)，北京：高等教育出版社，1990. 101 
2 Pasternack R F, Bustamante C, Collings P J, et al. Porphrin assemblies on DNA as studied by a resonace light- scattering technique. J Am Chem Soc, 1993, 115: 5393

3 Placek G. in Handbuch der Radiologie, Vol. VI. Marx E. (Akademische Verlagsgesellschaft, Leipzig, 1934), English Translation by A. Werbin: Rayleigh and Raman, UCRL Transl. No. 526L, Available from National Technical Information Service, US. Department of Commerce, Springfeild, Va. Part 2: 205 374

4 Bauer D R, Hudson B, Pecora R. Resonce enhance deploarized rayleigh scattering from diphenylpolyenes. J Chem Phys, 1975, 63: 588

5 Miller G A. Fluctuation theory of resonance enhancement of rayleigh scattering in absorbing media. J Phys Chem, 1978, 82: 616

6 Anglister J, Steinberg I Z. Depalarized rayleigh light scattering in absorption bands measured in lycopene solution. Chem Phys Lett, 1979, 65: 50

7 Anglister J, Steinberg I Z. Measurement of the depolarization ratio of rayleigh scattering at avsorption bands. Chem Phys Lett, 1981, 74: 786

8 Nelson W H, Howard W F, Pecora R. Use of ligand probes in light scattering 3 preresonance enhanced depolarized rayleigh scattering of dibenzoylmethane and selected diorganotin( v ) compleses. Inorg Chem, 1982, 21: 1483

9 Anglister J, Steinberg I Z. Resonance rayleigh scattering of cyanine dyes in solution. J Chem Phys, 1983, $78: 5358$

10 Magill J V, Clarke J H R. Photon correlation sepetroscopy using resonance enhanced light scattering. J Phys Chem, 1985, 89: 734

11 Chiarelio R, Reinisch L. Measurement of the resoncetly enhanced quasielastic light scattering cross sections of heme proteins. J Chem Phys, 1988, 88: 1253

12 Huang C Z, Li K A, Tong S Y. Determination of nucleic acids by a resonance light- scattering technique with $\alpha, \beta, \gamma, \&$ Tetrakis [4-(trimethylammoniumyl) phenyl] porphine. Anal Chem, 1996, 68 (13): 2259

13 Huang C Z, Li K A, Tong S Y. Determination of nanograms of nucleic acids by their enhancement effect on the resonance light scattering of the cobalt ( (E) /4-[ (5 chloro 2- pyridyl) azo]-1, 3-diaminobenzene complex. Anal Chem, 1997, 69: 514

14 Pasternack R F, Schaefer K F, Hambright P. Resonance light scattering studies of porphrin diacid aggregates. Inorg Chem, 1994, 33: 2062

15 Ma C Q, Li K A, Tong S Y. Microdetermination of proteins by resonance light scattering spetroscopy with bromophenol blue. Anal Biochem, 1996, 239(1): 86

16 Ma C Q, Li K A, Tong S Y. Rayleigh light scattering spectroscopy: application to the determination of proteins using bromophrogallol red. Bull Chem Soc Jpn, 1997, 70: 129

17 Ma C Q, Li K A, Tong S Y. Enhancement of rayleigh light scattering of acid chrome blue K by proteins and protein assay by the scattering technique. Analyst, 1997, 122: 361

18 Ma C Q, Li K A, Tong S Y. Microdetermination of proteins by resonance light scattering spectroscopy with tetraiodo phenol sulfonphthalein. Fresenius J Anal Chem, 1997, 357: 915

19 魏永巨, 李克安, 童沈阳. 蛋白质-铬天青 $\mathrm{S}$ 体系的弹性光散射及其初步分析应用. 化学学报, 1998, 56: 290

20 Ma C Q, Li K A, Tong S Y. Selective spectrophotometric determination of human serum albumin with tetraiodo phenol sulfonphthalein. Anal Lett, 1997, 30(4): 739

21 Pinnell A E, Northam B E. New automated dye-bing method for serum albumin determination with bromcresol purple. Clin Chem, 1978, 24: 80

22 De Paula J C, Robblee J H, Pasternack R F. Aggregation of chlorophyll a probed by resonance light scattering spectroscopy. Biophys J, 1995, 68: 335

23 刘绍璞, 刘忠芳, 李 明. 离子缔合物二级散射光谱的分析应用. 化学学报, 1995, 53: 1178

24 Liu S P, Liu Z F. Studies on the resonant luminescence spectra of rhodamine dyes and their iorr association complexes. Spectrochim Acta (Part A), 1995, 51: 1497

25 Pasternack R F, Collings P J. Resonance light scattering: a new technique for studying chromophore aggregation. Science, 1995, 269: 935

(1998_09_23) 